\title{
Multi-objective Optimization of Technical Stock Market Indicators using GAs
}

\author{
Magda B. Fayek \\ Computer Eng. Dpt., Faculty of \\ Eng., \\ Cairo Univ., Giza, Egypt
}

\author{
Hatem M. El-Boghdadi \\ Computer Eng. Dpt., Faculty of \\ Eng., \\ Cairo Univ., Giza, Egypt
}

\author{
Sherin M. Omran \\ Computer Eng. Dpt., Faculty of \\ Eng., \\ Cairo Univ., Giza, Egypt
}

\begin{abstract}
Recent financial researches showed that technical indicators are useful tools for stock prediction. Technical indicators are used to generate trading signals (buy/sell) signals. The main problem of an indicator usage is to determine its appropriate parameters. In this paper a new GA based technique for optimizing the parameters of a collection of technical indicators over two objective functions Sharpe ratio and annual profit is proposed. The technique handles four indicators DEMAC (Double Exponential Moving Average Crossovers), RSI (Relative Strength Index), MACD (Moving Average Convergence Divergence), and MARSI (Moving Average RSI) indicators. The technique was tested on 30 years of historical data of DJIA (Dow Jones Industrial Average) stock index. Results showed that the optimized parameters obtained by the proposed technique improved the profits obtained by the indicators with their typical parameters, the Buy and Hold strategy and the random strategy.
\end{abstract}

\section{General Terms}

Evolutionary Algorithms, Economics, Prediction.

\section{Keywords}

Technical Analysis, Genetic Algorithms, Parameter Optimization.

\section{INTRODUCTION}

Predicting the price movements in stock markets has been a major challenge for traders. The primary area of concern for any trader is to hit the ideal time to buy and sell. However, financial time-series is very noisy and volatile and hence very difficult to forecast. Errors in stock prediction may cause many losses, so stock prediction is always an important point of research.

Generally, there are three schools of thoughts concerning stock prediction. The first one is the (EMH) Efficient Market Hypothesis or the random walk theory which states that, stock market prices evolve according to a random walk so that past price movement cannot be used to predict its future movements $[1,2]$.

The second one is the fundamental analysis which is a method of evaluating securities by examining the characteristics and intrinsic value of a stock. It requires a large financial and accounting data set which is very hard to obtain and with low reliability [3].

Technical analysis represents the third view of the stock prediction. [3] Technical Analysis is the forecasting of future financial price movements based on an examination of past market actions. Technical analysis is based on the use of technical stock market indicators that are configured according to a set of parameters. These indicators are the mathematical calculations that day traders use in their charts in order to anticipate the price evolution. The complexity of the indicators may vary from one indicator to another. Some indicators are performed using simple formulae and are easy to understand while others use some complex structures and need a large number of input variables. The performance of the indicator does not necessarily depend on its complexity. Traders use indicators in two main ways: to confirm price movement, and to form buy and sell signals [3].

Technical indicators are classified as: leading indicators and lagging indicators. Examples of technical indicators are ROC (Rate Of Change), RSI (Relative Strength Index), Moving Averages, CCI (Commodity Channel Index) and OBV (On Balance Volume) indicators. The leading indicators generate signals preceding the price movement. They represent a form of price momentum over a fixed look-back period. Relative Strength Index (RSI) is one of the most popular leading indicators [4]. Lagging indicators are designed to follow the price action. They are commonly referred to as trendfollowing indicators. Examples of the lagging indicators are the moving averages [3]. There are some other indicators that are considered as leading indicators but with a bit of lag such as Moving Average Convergence Divergence (MACD) and Moving Average Relative Strength Index (MARSI) [5].

Attempts for Technical Indicators' optimization focused mainly on two main trends: Generating new trading rules using a collection of indicators and optimizing the parameters of indicators.

Hirabayashi et al. [6] used Genetic Algorithms to generate new trading rules for the Foreign Exchange market. SeungKyu Lee \& Byung-Ro Moon [7] developed a modular Genetic Programming to find attractive trading rules. They defined the attractive rule to be the one that is profitable, simple, and frequent.

Fernandez Blanco et al. [8] proposed an EA (Evolutionary Algorithm) based technique to optimize the parameters of the MACD (Moving Average Convergence Divergence) indicator. Both Adriano Simoes et al. [9] and V.Kapoor et al. [10] used Genetic Algorithms to optimize the parameters of SMAC indicator (Simple Moving Average Crossovers). 
The main contribution in this work is that GA is used for tuning the parameters of technical indicators over two objective functions annual profit and Sharpe ratio. As grown to our knowledge this is the first time these two objective functions are combined in such techniques. This means that GA is used to find the best collection of parameters for each indicator. The best parameters are the parameters that provide the best buy/sell signals which provide the highest profits. The work handled four different indicators Moving Average Convergence/Divergence (MACD), Double Exponential Moving Average crossovers (DEMAC), Relative Strength Index (RSI) indicator and finally, the Moving Average RSI indicator (MARSI). The performance of the optimized indicators is evaluated against the Buy and Hold strategy, the random strategy, and the indicators with their typical parameters.

The Buy and Hold is a passive investment strategy in which stocks are bought and held for a long period of time, regardless of the fluctuations in the market [11].

The next sections of the paper are organized as follows: a brief overview of Genetic Algorithms is described in section 2 , followed by an explanation of the selected indicators in section 3. Section 4 represents the proposed system and the empirical results are presented in section 5. Finally, conclusion is given in section 6 .

\section{GENETIC ALGORITHMS}

A genetic algorithm is a problem solving method that uses genetics as its model of problem solving. It's a search technique used to find near optimum solutions for optimization and search problems [12], [13].

Figure 1 shows the basic steps of Genetic Algorithms (GAs). First, a population of random solutions is provided. For each chromosome in the population fitness function is evaluated. The chromosomes with the highest fitness values are more probably to be selected for reproduction using crossover and mutation. If the stopping criteria are not met the procedure is repeated by evaluating again the new population and so on.

\section{BACKGROUND}

In this section we describe the indicators that are used in this paper. These indicators are DEMAC (Double Exponential Moving Average Crossovers), RSI (Relative Strength Index), MACD (Moving Average Convergence Divergence), and MARSI (Moving Average Relative Strength Index).

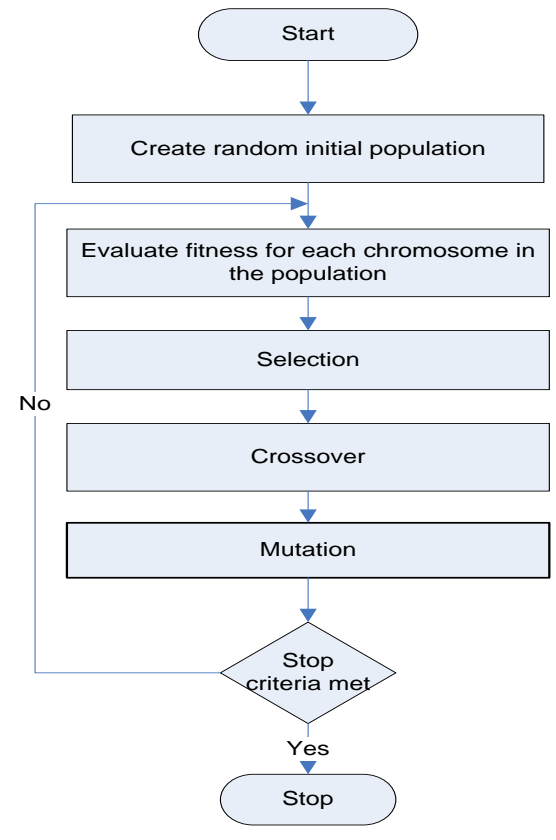

Figure 1: Genetic Algorithm flowchart

\subsection{DEMAC (Double Exponential Moving Average Crossovers)}

A moving average is the average value of data over a specified time period. The most common types of moving averages are the Simple Moving Average (SMA) and the Exponential Moving Average (EMA). The SMA is simply the average of closing prices of the last $n$ days. This average is moving because at the end of each trading day, the last day is added, while the earliest day of the previous average is dropped [10]. The problem with SMA is that all the trading days have the same weight. So fluctuations in the earlier days may affect the accuracy of the average. The SMA for a given stock could be described as follows.

$\operatorname{SMA}(i)=\frac{\sum_{i=1}^{n} \operatorname{price}(i)}{n}$

Where $n$ is the number of days for calculating the average and $\operatorname{price}(i)$ is the closing price for day $i$

The EMA assigns larger weight to the most recent day of calculation. This causes the EMA to follow the prices more closely most of the time as compared to SMA. The following equation calculates the EMA (Exponential Moving Average) for day $i$.

$$
\begin{aligned}
& \begin{array}{c}
E M A(i)=\text { weight }_{\text {current }} * \text { price }(i)+\text { weight }_{M A} * E M A(i \\
-1)
\end{array} \\
& \text { weight }_{\text {current }}=\frac{2}{n+1} \\
& \text { weight }_{M A}=100 \%-\text { weight }_{\text {current }}
\end{aligned}
$$


Where, $n$ is the number of days for calculating the average

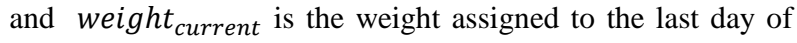
calculation (day $i$ ). And weight MA $_{\text {in }}$ is the weight assigned to the previous EMA.

In this paper trading with EMA is performed by using double EMA crossovers. A buy signal is generated when the shorter moving average (short term moving average) is crossed above the longer moving average (long term moving average) because this represents the beginning of an uptrend.

$E M A_{\text {short }}(i)>E M A_{\text {long }}(i)$

A sell signal is generated when the shorter moving average is crossed below the longer moving average because this represents the beginning of a down trend.

$E M A_{\text {short }}(i)<E M A_{\text {long }}(i)$

One of the famous combinations of moving averages is $20-50$ crossovers.

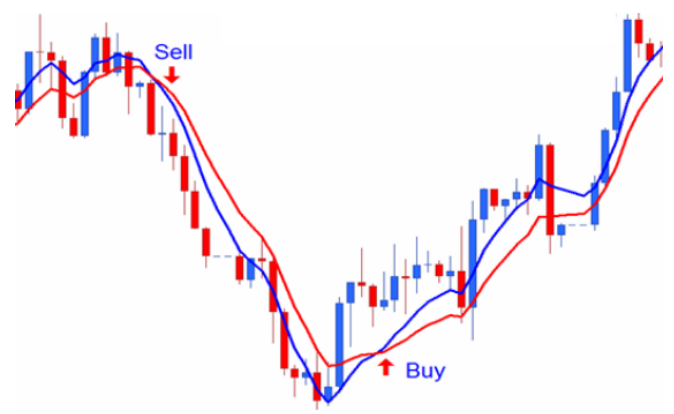

Figure 2 Double moving average crossovers

\subsection{MACD (MOVING AVERAGE CONVERGENCE/DIVERGENCE)}

MACD stands for Moving Average Convergence /Divergence. Convergence is the coming together of two or more indicators. Divergence is the moving apart of two or more indicators [14].

MACD is calculated by subtracting a long term EMA from a short term EMA and plotting a line graph of the difference called MACD line. Then calculating another EMA for that difference called the signal line.

The MACD formula is as follows:

$M A C D=E M A_{\text {short }}-E M A_{\text {long }}$

Signal $=E M A(M A C D)$

As seen from the last equation the signal line is calculated by taking the Exponential moving Average of the pre-calculated MACD line. A buy signal is generated when the MACD line crosses above the signal line or the MACD line crosses above through zero line. A sell signal is generated when the MACD line crosses below the signal line or crosses down through zero line. The typical parameters of the MACD indicator are $(12,26$, and 9 days). Where 12 and 26 are the short and the long EMA (Exponential Moving Averages) and 9 is the signal value.

\subsection{RSI (RELATIVE STRENGTH INDEX)}

RSI or Relative Strength Index [4] is a kind of momentum oscillators that is used to calculate the market recent gains against its recent losses and translates that information into a number between 0 and 100 [4].

$\mathrm{RS}=\frac{\text { Ups of } \mathrm{n} \text { days }}{\text { Downs of } \mathrm{n} \text { days }}$

$\mathrm{RSI}=100-\frac{100}{1+\mathrm{RS}}$

Where ups of $\mathrm{n}$ days are the total gains calculated over the last $\mathrm{n}$ days and the downs of $\mathrm{n}$ days are the total losses calculated over the last $\mathrm{n}$ days.

In the typical RSI, values above 70 indicate an overbought condition and hence a sell signal while values below 30 indicate oversold conditions and hence a buy signal.

The 30-70 levels are calculated by taking a constant shift value above and below the center line 50\% line (typically 20 ). The standard number of days used for calculating RSI is 14 days [4]. The RSI parameters to be optimized in our research are the number of days used as the look back period, the lower and the upper thresholds (bands).

Previous works that optimized RSI indicator used the number of days as the only parameter of RSI and calculated the lower and upper bands in a standard way [15]. In this work the GA learns to select the appropriate thresholds that determine the overbought and oversold conditions.

\subsection{MARSI (MOVING AVERAGE RSI)}

MARSI stands for Moving Average Relative Strength Index. MARSI is an indicator used to smooth out the action of RSI indicator. This indicator is the same a RSI except that we calculate a Simple Moving Average (SMA) for the calculated RSI indicator. Instead of buying or selling when RSI crosses the thresholds, Buy and sell signals are generated when the moving average crosses above or below the threshold levels.

$M A R S I=S M A(R S I)$

The parameters of the MARSI indicator are: The number of days for look-back period, the lower and upper thresholds and the number of days to average the RSI.

\section{THE PROPOSED ALGORITHM}

\subsection{Genetic Encoding}

In this paper GA is used for optimizing the parameters of four technical indicators in order to improve the trading profits.

These indicators are:

- DEMAC (Double Exponential Moving Averages Crossovers): The parameters optimized for the DEMAC are the $E M A_{\text {short }}$ and $E M A_{\text {long. }}$. 
- MACD (Moving Average Convergence Divergence): The parameters to be optimized for the MACD indicator are $E M A_{\text {short }}$ and $E M A_{\text {long }}$ and the signal line value.

- $\quad$ RSI (Relative Strength Index): The parameters to be optimized for the RSI indicator are $n$ which is the number of days used for calculating the RSI, the lower and the upper thresholds.

- MARSI (Moving Average Relative Strength Index): The parameters to be optimized for the MARSI are $n 1$ which is the number of days used for calculating the RSI, $n 2$ which is the number of days used for averaging the RSI, the lower and the upper thresholds.

Our GA chromosome is composed of four main parts with each part corresponding to one of the indicators to be optimized. Genes are real numbers representing the different parameters.

Table 1 : An example of a chromosome

\begin{tabular}{|c|c|c|c|c|c|c|c|c|c|c|c|}
\hline \multicolumn{2}{|c|}{ DEMAC } & \multicolumn{3}{|c|}{ MACD } & \multicolumn{3}{c|}{ RSI } & \multicolumn{4}{c|}{ MARSI } \\
\hline 20 & 50 & 12 & 26 & 9 & 14 & 30 & 70 & 14 & 30 & 70 & 14 \\
\hline
\end{tabular}

The first part consists of two genes that represent the two parameters of the DEMAC which are (EMA short and $\left.E M A_{\text {long }}\right)$ consecutively. The second part consists of three genes corresponding to the MACD indicator $E M A_{\text {short }}$, $E M A_{\text {long }}$ and signal.

The next three genes represent the three parameters of the RSI indicators which are the number of days used for RSI calculation, the lower threshold and the upper threshold.

The last block of genes (the last part) consists of four genes corresponding to the parameters of the MARSI indicator. The first three of them are the same as RSI while the fourth one represent the number of days used to average the RSI.

The numbers presented in table 1 are the typical parameters used for each indicator [15].

There are some boundary constraints used for each of the parameters under study:

DEMAC constraints:

- $\quad \mathrm{EMA}_{\text {short }}=[1,100]$

- $\quad \mathrm{EMA}_{\text {long }}=\left[\mathrm{EMA}_{\text {short }}, 200\right]$

The 100 and 200 days are selected from heuristics because value above 100 for the short moving average and values above 200 for the longer moving average do not lead to good solutions. These parameters are the well known parameters used for long term trading [3].

MACD constraints:

- $\quad$ Signal $<E M A_{-}$short $<E M A_{-}$long

- $\quad$ EMA_short $=[3,100]$

- $\quad$ EMA_long $=[$ EMA_short, 100]

- $\quad$ Signal $=[1$, EMA_short $]$
As mentioned from above these values are selected from heuristics. According to our experiments values above 100 lead to poorer solutions in the testing phase.

RSI constraints:

- $\quad$ N_RSI $=[3,100]$

- $\quad$ Lower boundary $=[10,40]$

- $\quad$ Upper_boundary $=[60,90]$

N_RSI is the number of days for calculating the RSI. As above, they are selected within the range from 3days to 100 days. The lower boundary (lower threshold) values are between 10 and 40 because values below 10 are rarely considered to provide buy signals. Values above 40 (from 40 to 50) represent a very high risk and may lead to poor solutions.

The upper boundary values are selected between 60 and 90 . As values below 60 do not lead to good solutions because sell signals are provided in the beginning of the trend. This leads to missing a large part of the profit. Values above 90 represent a very high risk because a very small amount of signals are provided and again this lead to missing profits.

MARSI constraints:

- $\quad$ N_RSI $=[3,100]$

- $\quad$ Lower boundary $=[10,40]$

- $\quad$ Upper_boundary $=[60,90]$

- $\quad$ N_SMA $=[1,100]$

The first three parameters are the same as RSI while the forth parameter is the number of days used for averaging the RSI. N_SMA is selected between 1 and 100. In case of 1 the MARSI is the same as RSI. Values above 100 make the indicator less sensitive to price changes.

\subsection{Parameters of the GA}

Preliminary experiments were performed to get the best parameters for GA. The finally selected parameters are those that are presented in the following table

Table 2 : GA parameters

\begin{tabular}{|l|l|}
\hline Population size & 100 \\
\hline $\begin{array}{l}\text { Number of } \\
\text { generations }\end{array}$ & 100 \\
\hline Selection technique & $\begin{array}{l}\text { Rank based } \\
\text { selection }\end{array}$ \\
\hline $\begin{array}{l}\text { Crossover } \\
\text { probability }\end{array}$ & 0.8 \\
\hline $\begin{array}{l}\text { Mutation } \\
\text { probability }\end{array}$ & 0.05 \\
\hline
\end{tabular}

The system is optimized over two objective functions explained in the next section called annual profit and Sharpe ratio. Vector Evaluated Genetic Algorithm (VEGA) has been adopted as the Multi-Objective Optimization (MOO) technique [16]. This approach is selected because it is easy to implement and computationally as efficient as single objective algorithms [16]. First, the population is divided into two subpopulations. Then selection is performed for each subpopulation over one of the objective functions. The subpopulations are recombined again for crossover and mutation. After a constant number of iterations (Every 5 iterations) an evaluation is performed using the weighted sum 
approach in order to prevent the solutions from converging entirely towards only one of the objectives.

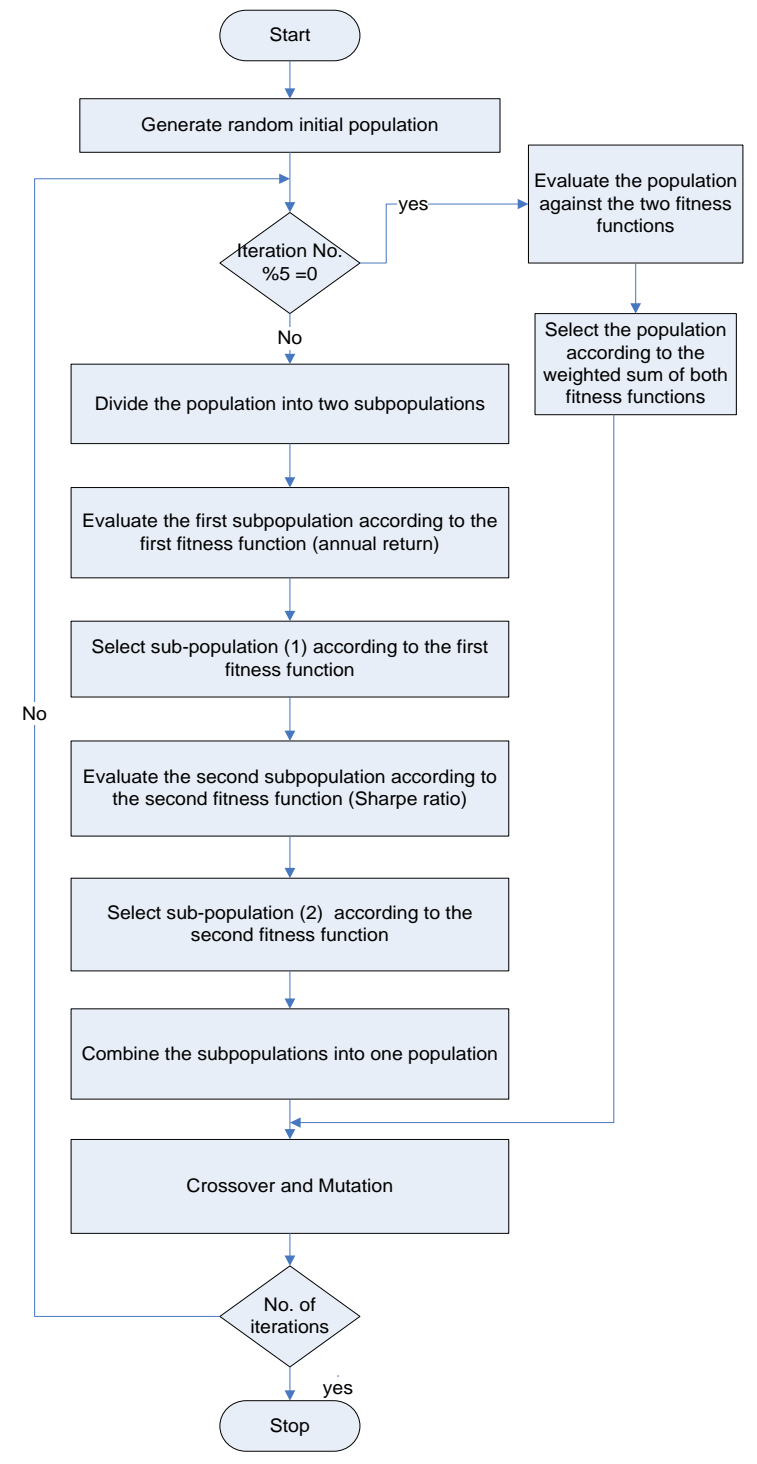

Figure 3: The proposed system flowchart

\subsection{The Fitness Function}

Chromosomes in each population are evaluated according to two objective functions, the annual return and the Sharpe ratio. The annual return is the yearly percentage profit, while the Sharpe ratio is an objective function used to calculate the mean return relative to the standard deviation of returns. These objective functions indicate two different criteria (profit and risk).

Annual return $=\left[\left(\frac{\text { return }}{\text { capital }}\right)^{\frac{1}{n}}-1\right] * 100$

$\mathrm{n}=$ number of trading days $/ 250$

Where $\mathrm{n}$ is the number of years and 250 is the maximum number of trading days per year.
The second fitness function is Sharpe ratio. This is a ratio developed by Nobel laureate William F. Sharpe to measure risk-adjusted performance [17]. The Sharpe ratio calculates the units of return per unit of risk. The ratio increases as the return increases or as the risk decreases.

Sharpe ratio $=\frac{\mu}{\sigma_{\mathrm{p}}}$

Where, $\mu$ is the mean value of all returns.

And $\sigma_{p}$ is the standard deviation of returns.

\subsection{The trading system}

In this research we focus on long term investment technique. Only in the beginning of the investment process an initial invested capital is infused into the market. No more money is infused during the investment period. This means that every trade affects all its followers. If the current trade is a profitable one, then the invested capital increases (profits are used for reinvestment in the next trades). If the current trade is a non profitable one, then the invested capital decreases which also affects the following trades.

In addition, trading is governed by the following rules: No two successive buying or selling signals are allowed (each buying signal is followed by a selling signal). The number of buys and sells are equal.

The advantage of this process is that it examines more firmly the capability of the trained parameters to hold on during all the investment periods.

\section{Experimental Results}

The proposed system is applied to the closing prices of DJIA (Dow Jones Industrial Average). It is the second oldest U.S market index which consists of 30 stocks from leading American industries. The optimized parameters are evolved on the data (From 1/1/1982 to 1/1/2012) 7564 trading days.

When optimizing the parameters, GA tends to make the highest returns in the training period. Sometimes GA as all other optimization techniques may suffer from over fitting and the obtained parameters may not provide any profits in other periods. So that, we split the series into training, validation, and testing periods in order to find robust solutions. We also used the rolling forward method [18] in which the data series is split into 7 sequences. Each sequence is represented as an independent run.

In each sequence the first 6 years are taken as training period, the next three years are taken as the validation period and the last three years are the testing period. The data in one sequence overlap the data in the next sequence. For each sequence we move all the periods three years forward. The data series is divided into 7 sequences called (A, B, C, D, E, $\mathrm{F}$, and $\mathrm{G})$. The second half of the training period with the entire validation period constitute the next training period. The testing period in one sequence become the validation period in the next sequence and so on. For example sequence 'A' training period starts from (1/1/1982) to (1/1/1988), 
validation period starts from (1/1/1988) to (1/1/1991), and testing period starts from (1/1/1991) to (1/1/1994). Sequence 'B' training period starts from (1/1/1985) to (1/1/1991), validation period starts from $(1 / 1 / 1991)$ to $(1 / 1 / 1994)$, and testing period starts from (1/1/1994) to (1/1/1997) and so on.

The evaluation period is divided into three different periods (training, validation, and testing periods). For each run the parameters are firstly optimized over the training period. The final population is reused as an initial population for the validation period. Then the final population of the validation period is re-evaluated against the training period and arranged according to the overall average gain of both training and validation periods. The best obtained parameters that match both the training and validation periods are then finally tested in the testing period.

\subsection{Buy and Hold strategy and typical indicators}

To test the performance of the work done many benchmarks have been taken into account. Firstly the obtained results are compared against the buy and hold strategy. This strategy implies to buy at the start of the investment period and sell at the end. The second is the indicators with their typical parameters. And finally, the results are compared against the random strategy.

We have implemented 50 independent runs for each of the previously mentioned sequences. For every 50 run we tabulated the best and the average of these runs. This process is repeated for all the indicators under study.

The results shown in the following tables are the average of the best results and the average of the average results for all the training, validation and testing periods over all indicators.

Table 3 shows the average performance of the DEMAC over all the 7 sequences. Averaging the results obtained for all training, validation, and testing periods.

As seen from the table the optimized DEMAC could beat the typical DEMAC in terms of return calculated by the first fitness function and risk calculated by the second fitness function (Sharpe ratio) for all the 3 periods for both the best and the average results. But it could not provide greater returns over the Buy and Hold strategy for all the three periods.
Table 3: The average performance of the DEMAC indicator over all the training, validation and testing periods

\begin{tabular}{|c|c|c|c|c|c|}
\hline & & Best & Average & Typical & $\mathbf{B} \& \mathbf{H}$ \\
\hline \multirow{2}{*}{ Train } & $\begin{array}{l}\text { Return } \\
(\%)\end{array}$ & 8.038 & 8.141 & 5.4488 & 11.176 \\
\hline & Sharpe & 2.997 & 2.446 & 0.2443 & ------ \\
\hline \multirow{2}{*}{ Valid } & $\begin{array}{l}\text { Return } \\
(\%)\end{array}$ & 7.286 & 6.399 & 1.7620 & 7.6275 \\
\hline & Sharpe & 0.535 & 0.211 & -0.0792 & ------ \\
\hline \multirow{2}{*}{ Test } & $\begin{array}{l}\text { Return } \\
\text { (\%) }\end{array}$ & 4.895 & 2.153 & 2.4373 & 7.8006 \\
\hline & Sharpe & 0.547 & 0.0461 & 0.0097 & ------ \\
\hline
\end{tabular}

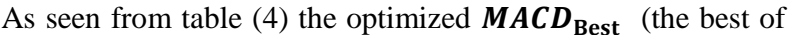
the 50 runs) and $\boldsymbol{M} \boldsymbol{A} \boldsymbol{C} \boldsymbol{D}_{\text {avg }}$ (the average of all points obtained from the 50 runs) parameters provided the best return and Sharpe ratios over the typical MACD in all the training, validation and testing periods. Both $\boldsymbol{M} \boldsymbol{A C} \boldsymbol{D}_{\text {Best }}$ and $\boldsymbol{M A C D}_{\text {avg }}$ outperformed the market in the average results of the validation periods where $\boldsymbol{M} \boldsymbol{A} \boldsymbol{C} \boldsymbol{D}_{\mathrm{Best}}$ provided an annual return of $9.795 \%$ and $\boldsymbol{M} \boldsymbol{A C} \boldsymbol{D}_{\text {avg }}$ provided an annual return of 9.67415\% while the Buy and Hold strategy provided $7.627 \%$. But they could not beat the market in both training and testing periods.

Table 4: The average performance of the MACD indicator over all the training, validation and testing periods

\begin{tabular}{|l|l|l|l|l|l|}
\hline \multicolumn{2}{|c}{ Train } & Best & Average & Typical & B\&H \\
\hline \multirow{2}{*}{ Valid } & $\begin{array}{l}\text { Return } \\
(\%)\end{array}$ & 9.829 & 8.366 & 4.5542 & 11.176 \\
\cline { 2 - 6 } & Sharpe & 0.205 & 0.19082 & 0.1449 & ------ \\
\hline \multirow{2}{*}{ Test } & $\begin{array}{l}\text { Return } \\
(\%)\end{array}$ & 9.795 & 9.67415 & 2.5424 & 7.627 \\
\cline { 2 - 6 } & Sharpe & 0.205 & 0.32241 & 0.1029 & ------ \\
\cline { 2 - 6 } & $\begin{array}{l}\text { Return } \\
\text { (\%) }\end{array}$ & 7.414 & 5.35158 & 3.3632 & 7.800 \\
\hline
\end{tabular}

Table 5: The average performance of the RSI indicator over all the training, validation and testing periods

\begin{tabular}{|c|c|c|c|c|c|}
\hline \multicolumn{2}{|c|}{} & Best & Average & Typical & B\&H \\
\hline \multirow{2}{*}{ Train } & $\begin{array}{c}\text { Return } \\
(\%)\end{array}$ & 11.054 & 10.295 & 5.367 & 11.176 \\
\cline { 2 - 6 } & Sharpe & 2.2156 & 2.251 & 2.059 & ------ \\
\hline \multirow{3}{*}{ Valid } & $\begin{array}{c}\text { Return } \\
(\%)\end{array}$ & 11.505 & 11.631 & 6.855 & 7.6275 \\
\cline { 2 - 6 } & Sharpe & 2.6276 & 2.429 & 2.339 & ------ \\
\hline \multirow{2}{*}{ Test } & $\begin{array}{c}\text { Return } \\
(\%)\end{array}$ & 9.3447 & 7.856 & 5.960 & 7.8006 \\
\cline { 2 - 7 } & Sharpe & 1.5505 & 2.145 & 2.015 & ------ \\
\hline
\end{tabular}


As shown in table (5) $\mathrm{RSI}_{\text {Best }}$ and $\mathrm{RSI}_{\mathrm{avg}}$ provided the highest returns over the other techniques in all periods. For example in the testing period the $\mathrm{RSI}_{\text {Best }}$ return is $9.3447 \%$ and $\mathrm{RSI}_{\mathrm{avg}}$ return is $7.8558 \%$ while the typical RSI return is $5.9607 \%$ and the Buy and Hold return is $7.8006 \%$.

Table 6 shows the results of the MARSI indicator. As seen from the table $M A R S I_{B E S T}$ gained higher returns than the other two benchmarks in all periods. MARSI avg provided higher returns than the typical MARSI in all the training, validation and testing periods. It also provided higher returns than the Buy and Hold strategy in two of the three periods. As seen from the table the MARSI $I_{\text {avg }}$ provided $12.4433 \%$ in the training period while the Buy and Hold provided $11.176 \%$. In the validation period the $M A R S I_{\text {avg }}$ return is $11.9921 \%$ while the Buy and Hold return is $7.6275 \%$. For the testing period the MARSI $I_{\text {avg }}$ return is $6.9391 \%$ while the Buy and Hold is $7.8006 \%$.

Table 6: The average performance of the MARSI indicator over all training, validation and testing periods

\begin{tabular}{|c|c|c|c|c|c|}
\hline \multicolumn{2}{|c|}{} & Best & Average & Typical & B\&H \\
\hline \multirow{2}{*}{$\begin{array}{c}\text { Trai } \\
\text { n }\end{array}$} & $\begin{array}{c}\text { Return } \\
(\%)\end{array}$ & 13.031 & 12.4433 & 4.3986 & 11.176 \\
\cline { 2 - 6 } & Sharpe & 1.3272 & 1.3195 & 1.9403 & ------ \\
\hline \multirow{2}{*}{$\begin{array}{c}\text { Vali } \\
\text { d }\end{array}$} & $\begin{array}{c}\text { Return } \\
(\%)\end{array}$ & 14.019 & 11.9921 & 6.5098 & 7.6275 \\
\cline { 2 - 6 } & Sharpe & 2.1625 & 4.1987 & 1.8402 & ------ \\
\hline \multirow{2}{*}{ Test } & $\begin{array}{c}\text { Return } \\
(\%)\end{array}$ & 8.4436 & 6.9391 & 6.2986 & 7.8006 \\
\cline { 2 - 6 } & Sharpe & 1.0582 & 1.5811 & 2.0019 & ------ \\
\hline
\end{tabular}

As shown from the tables, the optimized parameters could beat the indicators with their typical parameters for all indicators over all periods.

RSI, and MARSI indicators could beat the buy and hold strategy or at least give equal performance to it for all periods. MACD indicator outperformed the Buy and Hold strategy only for one of the three periods. DEMAC indicator was the only indicator that could not beat the buy and hold strategy. This is because the DEMAC is a lagging indicator that is more suitable for trending periods than trading periods (periods of sideway). Data in the DJIA index is volatile most of the time and there are long periods of sideways which reduce the performance of the DEMAC indicator.

\subsection{Random strategy}

To compare with the random strategy, as done before, 50 independent runs for the random strategy have been executed.

For each run we calculated the annual return and the Sharpe ratio. Then, obtained results are averaged for all training, validation and testing periods. Figure 2 shows the average annual returns gained by using the random strategy vs. the annual returns gained by the optimized indicators. Figure 3 points out the average Sharpe ratio for the random strategy vs. the optimized indicators.

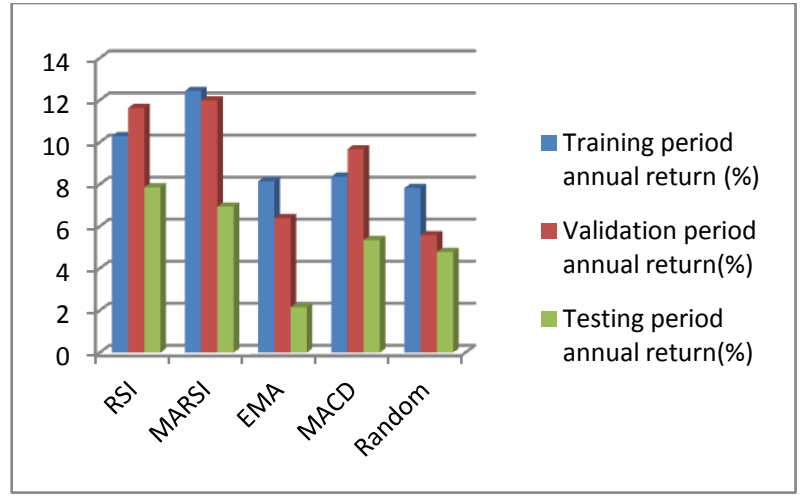

Figure 4: Average annual return for the optimized indicators vs. the random strategy

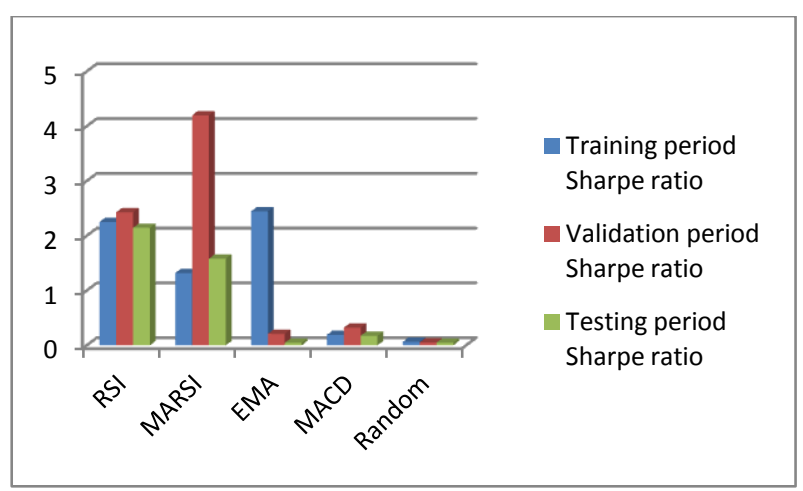

Figure 5: Average Sharpe ratio for the optimized indicators vs. the random strategy

\section{CONCLUSIONS}

This paper presented a MOGA (Multi-objective Genetic Algorithm) technique to optimize the parameters of four different technical indicators. The objectives under study are the annual profit and the Sharpe ratio. The proposed system is applied to 30 years of the closing prices of the DJIA (Dow Jones Industrial Average) stock index. The data series is split into training, validation and testing periods. We also used the rolling forward method in which each training period is 6 years long, every validation period is 3 years long, and every testing period is 3 years long. Results showed that the optimized parameters could beat the indicators with their typical parameters for all indicators over all sequences. Three of the indicators under study (RSI, MACD, and MARSI) could outperform the market (Buy and Hold) most of the time. DEMAC was the only indicator that could not beat the market that's because of the nature of the moving average indicators as lagging indicators. Comparisons with random strategy also proved that optimized indicators could beat the random strategy for all the training, validation periods and most of the testing periods.

\section{REFERENCES}

[1] S. Taylor. (1986). "Modeling Financial Time Series", John Wiley \& Sons

[2] Malkiel, Burton G. (1973). A Random Walk Down Wall Street (6th ed.). W.W. Norton \& Company, Inc. ISBN 0393-06245-7

[3] John J. Murphy. TECHNICAL ANALYSIS OF THE FINANCIAL MARKETS (A COMPREHENSIVE 
GUIDE TO TRADING METHODS AND APPLICATIONS). New York Institute of Finance 1999.

[4] Teeples, Allan W., "An Evolutionary Approach to Optimization of Compound Stock Trading Indicators Used to Confirm Buy Signals"(2010). All Graduate Thesis and Dissertations. Paper 820.

[5] M.A.H. Dempster and Chris M. Jones. (2000). The profitability of intra-day FX trading using technical indicators. University of Cambridge Judge Institute of Management Studies.Research Papers, No.35/2000. Cambridge: University of Cambridge.

[6] Akinori Hirabayashi, Claus Aranha and Hitoshi Iba. Optimization of the Trading Rule in Foreign Exchange using Genetic Algorithm. In GECCO 2009.

[7] Seung-kyu Lee and Byungo-Ro Moon. Finding attractive rules in stock markets using a modular genetic programming. In GECCO 2009.

[8] P.Fernandez, D.Bodas, F.Soltero and J.I.Hidalgo .Technical market indicators optimization using EA. In GECCO 2008.

[9] Adriano Simões, Rui Ferreira Neves, Nuno Horta . An Innovative GA Optimized Investment Strategy based on a New Technical Indicator using Multiple MAS. In proceeding of: ICEC 2010 - Proceedings of the International Conference on Evolutionary Computation, [part of the International Joint Conference on Computational Intelligence IJCCI 2010], Valencia, Spain, October 24 - 26, 2010.

[10] V.Kapoor, S.Dey, and A.P Khurana. Genetic Algorithm: An Application to Technical Trading System Design. International Journal of Computer Applications (0975 8887), Volume 36- No.5, December 2011.
[11] Devayan Mallick, Vincent C.S. Lee and Yew Soon Ong. An Empirical Study of Genetic Programming Generated Trading Rules in Computerized Stock Trading Service System. In IEEE proceedings. (2008).

[12] S.N.Sivanandam \& S.N.Deepa . Introduction to Genetic Algorithms. Springer 2008.

[13] Darrell Whitley. A Genetic Algorithm Tutorial. Statistics and Computing (1994) 4, 65-85.

[14] Gerald Appel. Financial Times Prentice Hall. 2005 Pearson Education, Inc.

[15] Diego J Bodas-Sagi , Pablo.Fernandez , J.lgnacio, Francisco J. Soltero and Jose L.Risco-Martin. Multiobjective optimization of technical market indicators. In GECCO 2009.

[16] Abdullah Konaka,_, David W. Coitb, Alice E. Smithc. Multi-objective optimization using genetic algorithms: A tutorial. In Reliability Engineering and System Safety 91 (2006) 992-1007 (ELSEVIER).

[17] Mark Choey and Andreas S. Weigend. Nonlinear Trading Models Through Sharpe Ratio Maximization. Leonard N. Stern School of Business, New York University. In: Decision Technologies for Financial Engineering (Proceedings of the Fourth International Conference on Neural Networks in the Capital Markets, NNCM-96), pp. 3-22.

[18] Lohpetch, D, David Corne. Multiobjective algorithms for financial trading: Multiobjective out-trades singleobjective. In proceeding of: Evolutionary Computation (CEC), 2011 IEEE Congress on 